doi: $10.15407 /$ ujpe62.02.0112

D.O. PLUTENKO, ${ }^{1,2}$ M.V. VASNETSOV ${ }^{1}$

${ }^{1}$ Institute of Physics, Nat. Acad. of Sci. of Ukraine

(46, Prosp. Nauky, Kyiv 03028, Ukraine)

2 Physical Engineering Teaching Research Center of Nat. Acad. of Sci. of Ukraine

(36, Academician Vernadsky Prosp., Kyiv 03680, Ukraine; e-mail: dmplutenko@gmail.com)

\title{
SYMMETRY IN A SPHERICAL-PARTICLE LIGHT SCATTERING AND A PHASE SHIFT INDUCED BY A PARTICLE TRANSLATION
}

\begin{abstract}
Diffraction of a linearly polarized plane wave and two counterpropagating orthogonally polarized plane waves by a spherical particle is considered. The influence of the particle translation on the far-field complex amplitude of the scattered wave is discussed. The possibility is shown to select symmetric directions of observation, in which the phase shift of a scattered wave depends the only on particle coordinate. All conclusions are generic as based on a symmetry only. The results can be useful for a new direct-measurement particle position detector engineering with high sensitivity and speed of response.

Keywords: Mie theory, light scattering, symmetries, particle position detector.
\end{abstract}

\section{Introduction}

The concept of symmetry in physics is used extremely widely for the explanation of different phenomena and the understanding of the general principles of the Nature. For instance, in spectroscopy, it is exploited for the classification of optical spectra of atoms, ions, molecules [1], and quantum dots [2]. Under some circumstances, the application of symmetry rules allows one to achieve a proper solution without complicated derivations. In the present work, we are going to demonstrate the issue as applied to the problem of a plane wave scattering by an isotropic sphere. Within this approach, we will describe some general relationships in the far field in the case of a translation of the object on which the wave is scattered.

The problem of the scattering of a plane electromagnetic wave by a homogeneous isotropic sphere is well known and finds the solution within the LorentzMie theory [3], which is based on expanding the in-

(C) D.O. PLUTENKO, M.V. VASNETSOV, 2017

112 cident and scattered waves in infinite series of spherical harmonics. Later, this theory was generalized to beams of arbitrary shape, and it is known as the generalized Lorentz-Mie theory (GLMT) [4, 5]. Despite the pioneering works appeared more than one hundred years ago, the real applications became possible only with the advent of large digital computers. For the historical review, algorithms, and approaches, see [6]. Nowadays, GLMT is widely used owing to the exploitation of microbeads in various areas, for example, an ultralow-threshold Raman laser can be based on dielectric microbeads [5], photonic nanojets are formed when a plane wave is diffracted by a dielectric microbead [7-9], etc. Another reason is that the spherical GLMT model can be the first-order approximation for the theory of scattering by nonspherical objects.

Experimental measurements corroborate the theory. A number of experimental methods are available for the detection of the field intensity near a sphere $[10,11]$, but much easier is the determination of the far-field intensity distribution. In theoretical

ISSN 2071-0194. Ukr. J. Phys. 2017. Vol. 62, No. 2 
studies, most approaches require the direct calculation of the electromagnetic field amplitude. The final result depends on the ratio of the wavelength to the size of a microbead, its material, and the material of an ambient medium. However, these factors do not influence the symmetry. According to the Mie theory, the scattered wave is invariant with respect to the same elements of the group of distance-preserving transformations $O(3)$ (with fixed point at the origin), as an incident wave for any homogeneous isotropic sphere and medium [3]. Exactly this fact is used in our study, and the result has been proved to be general. Some general statements for optical forces [12] and the formation of nanojets $[13,14]$ were already proved, by using symmetry considerations. In the present work, we consider some important relationships for the far-field scattering in specific directions related to the symmetry of the geometrical configuration with account for the polarization (linear) of an incident wave. The idea is to demonstrate that the phase shift between the waves scattered in such determined directions depends only on the particle position in the case of Rayleigh scattering or arbitrary-size spherical particle.

To the best of our knowledge, this approach was not considered before. We believe that the results obtained are of practical interest in a new-type particleposition detector engineering.

\section{Theoretical Considerations}

The electric field $\mathcal{E}(\boldsymbol{r}, t)$ component of a monochromatic electromagnetic wave can be expressed via the complex amplitude as $\boldsymbol{E}(\boldsymbol{r})$

$\mathcal{E}(\boldsymbol{r}, t)=\frac{1}{2}\left[\boldsymbol{E}(\boldsymbol{r}) \exp (-\mathrm{i} \omega t)+\boldsymbol{E}^{*}(\boldsymbol{r}) \exp (\mathrm{i} \omega t)\right]$,

where $\omega$ is the frequency of oscillations, $t$ is the time, and $\boldsymbol{r}$ is the coordinate vector. For convenience, we use $\boldsymbol{E}(\boldsymbol{r})$ as the representation of the wave itself $\mathcal{E}(\boldsymbol{r}, t)$. In the case of linear isotropic medium, the vector $\boldsymbol{E}(\boldsymbol{r})$ satisfies the Helmholtz equation $\left[\Delta+k^{2}\right] \boldsymbol{E}(\boldsymbol{r})=0$ with the additional condition $\operatorname{div} \boldsymbol{E}(\boldsymbol{r})=0$, which indicates the transverse nature of the electromagnetic wave, $k$ is the wavenumber $k=\sqrt{\varepsilon \mu} \omega / c, \varepsilon$ and $\mu$ are the dielectric permittivity and magnetic permeability, and $c$ is the speed of light.

In the case of the monochromatic wave scattering on a particle, the complex amplitude $\boldsymbol{E}(\boldsymbol{r})$ in the region outside the particle can be expressed as a sum of the incident $\boldsymbol{E}^{\mathrm{i}}(\boldsymbol{r})$ and scattered $\boldsymbol{E}^{\mathrm{s}}(\boldsymbol{r})$ waves [15].
The last one will be mainly discussed in the present work. In the case of isotropic homogeneous medium, the far-field complex amplitude of this constituent has the form

$\boldsymbol{E}^{\mathrm{s}}(\boldsymbol{r})=\frac{1}{r} \exp (\mathrm{i} k r) \boldsymbol{F}(\boldsymbol{n})$,

where $\boldsymbol{n}=\boldsymbol{r} / r$ defines direction of observation, $r=$ $=|\boldsymbol{r}|$, and $\boldsymbol{F}(\boldsymbol{n})$ describes the far-field complex amplitude distribution. Unlike $\boldsymbol{E}^{\mathrm{s}}(\boldsymbol{r})$, the far-field pattern $\boldsymbol{F}(\boldsymbol{n})$ is a function only of the observation angles, and it is independent of $r$. In general, it has no other limitations except $\boldsymbol{F}(\boldsymbol{n}) \perp \boldsymbol{n}$, which occurs due to the transverse nature of electromagnetic waves.

In the case of particle illumination by an arbitrarily shaped monochromatic wave (e.g., the HermiteGauss mode [16]), it is necessary in most cases to recalculate the far-field distribution for any new position of the particle. In general, there is no obvious relationship between far-field complex amplitude distributions obtained during the scattering on a particle placed at different positions. When the particle is illuminated by a plane wave, the particle translation changes only the phase in the far field, while the amplitude and polarization remain the same. To prove this statement, let us consider firstly the simultaneous translation of the particle and the incident plane wave by the same vector $\boldsymbol{R}$ from the origin. According to 2 , the phase shift in this case amounts to $-k \boldsymbol{R} \boldsymbol{n}$. The phase shift implemented to the incident wave,

$\boldsymbol{E}_{p w}(\boldsymbol{r})=\boldsymbol{E}_{0} \exp \left(\mathrm{i} k \boldsymbol{n}_{\mathrm{pw}} \boldsymbol{r}\right)$,

is $-k \boldsymbol{R} \boldsymbol{n}_{\mathrm{pw}}$, where $\boldsymbol{n}_{\mathrm{pw}}$ defines the plane wave propagation direction. Therefore, the far-field phase shift $\triangle \varphi$ in the case of particle translation is

$\triangle \varphi(\boldsymbol{n}, \boldsymbol{R})=k\left[\boldsymbol{n}_{\mathrm{pw}}-\boldsymbol{n}\right] \boldsymbol{R}$.

Therefore,

$\boldsymbol{F}(\boldsymbol{n}, \boldsymbol{R})=\exp (\mathrm{i} \triangle \varphi(\boldsymbol{n}, \boldsymbol{R})) \boldsymbol{F}_{0}(\boldsymbol{n})$,

where $\boldsymbol{F}_{0}(\boldsymbol{n})$ defines the far-field amplitude, when the scattering particle is at the origin. In this way, we describe the far field of a scattered wave not only as a function of the scattering direction $\boldsymbol{n}$, but also as a function of the particle position $\boldsymbol{R}$.

\subsection{Phase relations \\ in the far field in the case of plane wave diffraction on a spherical particle}

In this work, the main object of consideration is the phase relationships between the vector function 


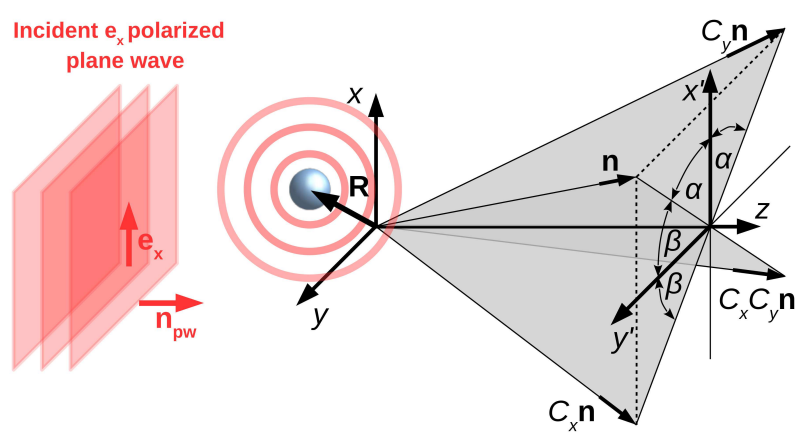

Fig. 1. Plane wave is scattered on the particle, $\boldsymbol{n}_{\mathrm{pw}}$ defines the direction of plane wave propagation, $\boldsymbol{n}_{\mathrm{pw}}=\boldsymbol{e}_{z}, \cos \alpha=$ $=n_{x}\left(n_{x}^{2}+n_{y}^{2}\right)^{-\frac{1}{2}}, \cos \beta=n_{y}\left(n_{x}^{2}+n_{y}^{2}\right)^{-\frac{1}{2}} ; \boldsymbol{n}$ is a unit vector which defines the main direction of observation. Vectors $C_{x} \boldsymbol{n}$ and $C_{y} \boldsymbol{n}$ are the mirror reflections of $\boldsymbol{n}$ from the $y z$ and $x z$ planes, respectively. $C_{x} C_{y} \boldsymbol{n}$ and $\boldsymbol{n}$ are symmetric to each other relative to the $z$ axis. $\boldsymbol{R}$ denotes a particle position with respect to the origin

$\boldsymbol{F}(\boldsymbol{n}, \boldsymbol{R})$ along two specific scattering directions in the case of incident linearly polarized plane wave and scattering particle with spherical symmetry. Without loss of generality, we assume that the incident wave is polarized along the $x$ axis, and the direction of propagation is $\boldsymbol{e}_{z}\left(\boldsymbol{n}_{\mathrm{pw}}=\boldsymbol{e}_{z}\right)$ (see Fig. 1). The incident wave complex amplitude can be expressed as

$\boldsymbol{E}^{\mathrm{i}}(\boldsymbol{r})=E_{0} \boldsymbol{e}_{x} \exp \left(\mathrm{i} k \boldsymbol{e}_{z} \boldsymbol{r}\right)$.

Such field has the symmetries

$C_{x} \boldsymbol{E}^{\mathrm{i}}\left(C_{x} \boldsymbol{r}\right)=-\boldsymbol{E}^{\mathrm{i}}(\boldsymbol{r})$,

$C_{y} \boldsymbol{E}^{\mathrm{i}}\left(C_{y} \boldsymbol{r}\right)=\boldsymbol{E}^{\mathrm{i}}(\boldsymbol{r})$,

where $C_{x}$ and $C_{y}$ are orthogonal matrices, which define reflections with respect to the plane $y z$ and $x z$, respectively. They have the form

$C_{x}=\left(\begin{array}{ccc}-1 & 0 & 0 \\ 0 & 1 & 0 \\ 0 & 0 & 1\end{array}\right)$,

$C_{y}=\left(\begin{array}{ccc}1 & 0 & 0 \\ 0 & -1 & 0 \\ 0 & 0 & 1\end{array}\right)$,

$\boldsymbol{F}_{0}(\boldsymbol{n})$ has the same symmetries as an incident plane wave with account for its polarization:

$C_{x} \boldsymbol{F}_{0}\left(C_{x} \boldsymbol{n}\right)=-\boldsymbol{F}_{0}(\boldsymbol{n})$,

$C_{y} \boldsymbol{F}_{0}\left(C_{y} \boldsymbol{n}\right)=\boldsymbol{F}_{0}(\boldsymbol{n})$.
Statements (11) and (12) are the particular results of the Mie theory. For example, they are shown in [3] for a spherical particle, but they can be also easily extended for any particle with spherical symmetry. Such symmetries occur only if the particle is located at the origin, while particle translation destroys them.

Let us consider the relation between $\boldsymbol{F}(\boldsymbol{n}, \boldsymbol{R})$, $\boldsymbol{F}\left(C_{x} \boldsymbol{n}, \boldsymbol{R}\right)$, and $\boldsymbol{F}\left(C_{y} \boldsymbol{n}, \boldsymbol{R}\right)$, as shown in Fig. 1, where $\boldsymbol{n}$ can be an arbitrarily chosen direction of observation, and $C_{x} \boldsymbol{n}$ and $C_{y} \boldsymbol{n}$ are reflections of the unit vector $\boldsymbol{n}$ corresponding to the incident wave symmetries.

In view of (4), (5), and (11), we have the expression

$\boldsymbol{F}\left(C_{x} \boldsymbol{n}, \boldsymbol{R}\right)=-\exp \left(2 \mathrm{i} k n_{x} R_{x}\right) C_{x} \boldsymbol{F}(\boldsymbol{n}, \boldsymbol{R})$.

For $C_{y}$, we have

$\boldsymbol{F}\left(C_{y} \boldsymbol{n}, \boldsymbol{R}\right)=\exp \left(2 \mathrm{i} k n_{y} R_{y}\right) C_{y} \boldsymbol{F}(\boldsymbol{n}, \boldsymbol{R})$,

and

$\boldsymbol{F}\left(C_{x} C_{y} \boldsymbol{n}, \boldsymbol{R}\right)=$

$=-\exp \left(2 \mathrm{i} k\left[n_{x} R_{x}+n_{y} R_{y}\right]\right) C_{x} C_{y} \boldsymbol{F}(\boldsymbol{n}, \boldsymbol{R})$,

where the unit vectors $C_{x} C_{y} \boldsymbol{n}$ and $\boldsymbol{n}$ are symmetric to each other relative to the $z$ axis.

Considering the far field in the scattering directions $\boldsymbol{n}, C_{x} \boldsymbol{n}, C_{y} \boldsymbol{n}$, and $C_{x} C_{y} \boldsymbol{n}$, we can see that their amplitudes are equal, and the polarizations are easily expressed through one another. Moreover, the far-field components along $\boldsymbol{e}_{x}$ are the same, and the components along $\boldsymbol{e}_{y}$ are the same or with opposite directions, as well as along $\boldsymbol{e}_{z}$.

The most interesting are the phase relations. The far-field phase difference occurring between the complex far-field amplitudes in the directions $\boldsymbol{n}$ and $C_{x} \boldsymbol{n}$ neither depends on the size of a particle and its material nor on a displacement of the particle along the $y$ and $z$ axes $\left(R_{y}\right.$ and $\left.R_{z}\right)$. The actual phase shift depends only on $R_{x}$ linearly and the projection of the $\boldsymbol{n}$ onto the $x$ axis $\left(n_{x}\right)$. The same occurs for $\boldsymbol{n}$ and $C_{y} \boldsymbol{n}$ directions, and the phase difference depends on $R_{y}$ and $n_{y}$ only, as it follows from (14). This phenomenon can be used for the determination of the particle position in the $x$ and $y$ directions.

However, because a phase is determined within $0 \div 2 \pi$ range, the coordinates are indistinguishable with projections $R_{x}$ and $R_{x}+\pi\left(k n_{x}\right)^{-1}$ on the $x$ axis in the case of a selected pair of observation directions $\left\{\boldsymbol{n}, C_{x} \boldsymbol{n}\right\}$. It can be fixed, by using more 
measurement directions. For example, let us consider two pairs of observation directions $\left\{\boldsymbol{n}_{1}, C_{x} \boldsymbol{n}_{1}\right\}$ and $\left\{\boldsymbol{n}_{2}, C_{x} \boldsymbol{n}_{2}\right\}$. Suppose that, for relatively prime integers $M_{1}$ and $M_{2}$, we have the equality

$\delta=\frac{\left|n_{1 x}\right|}{M_{1}}=\frac{\left|n_{2 x}\right|}{M_{2}}$,

where $n_{1 x}=\boldsymbol{n}_{1} \boldsymbol{e}_{x}$ and $n_{2 x}=\boldsymbol{n}_{2} \boldsymbol{e}_{x}$. In this case, the particle position along the $x$ axis is measurable in the $\left[-\pi(2 k \delta)^{-1}, \pi(2 k \delta)^{-1}\right)$ interval, and it is impossible to recognize a difference between the particle position projections onto the $x$ axis, which equal $R_{x}$ and $R_{x}+\pi(k \delta)^{-1}$. Relation (16) can be extended for numerous pairs of observation directions

$\delta=\frac{\left|n_{1 x}\right|}{M_{1}}=\frac{\left|n_{2 x}\right|}{M_{2}}=\ldots=\frac{\left|n_{N x}\right|}{M_{N}}$.

In this case, $M_{1}, M_{2}, \ldots, M_{N}$ do not have a common divisor. In theory, to be able to determine $R_{x}$ of a particle along the whole real axis, two pairs of measurement directions $\left\{\boldsymbol{n}_{1}, C_{x} \boldsymbol{n}_{1}\right\}$ and $\left\{\boldsymbol{n}_{2}, C_{x} \boldsymbol{n}_{2}\right\}$ are sufficient in the case where $n_{x 1} / n_{x 2}$ is an irrational number. In practice, it is not so, because of the measurement sensibility and the precision of measurement direction selection. Anyway, the area of unambiguous particle $R_{x}$ position detection can be expanded, by increasing the number of pairs of measurement directions $\left\{\boldsymbol{n}_{k}, C_{x} \boldsymbol{n}_{k}\right\}$. Of course, the sensibility of a measurement is increased as well. The same is true for the measurement of the projection of a particle position $\boldsymbol{R}$ onto the $y$ axis. This means that the transversal coordinates of a particle $R_{x}$ and $R_{y}$ can be measured except the longitudinal displacement $R_{z}$. To overcome this problem, the scattering of two counterpropagating orthogonally polarized plane waves on a particle can be used.

\subsection{Phase relations in the far field in the case of diffraction of two counter-propagating orthogonally polarized plane waves on a spherical particle}

In the case of one plane wave illuminating a particle, we can select the directions, the phase difference between which in the far field depends neither on $R_{z}$ nor the size and material of the scattering particle, but only on the particle displacement along the $x$ or $y$ axis $\left(R_{x}\right.$ or $\left.R_{y}\right)$. Let us show that, in the case of two counter-propagating orthogonally polarized plane

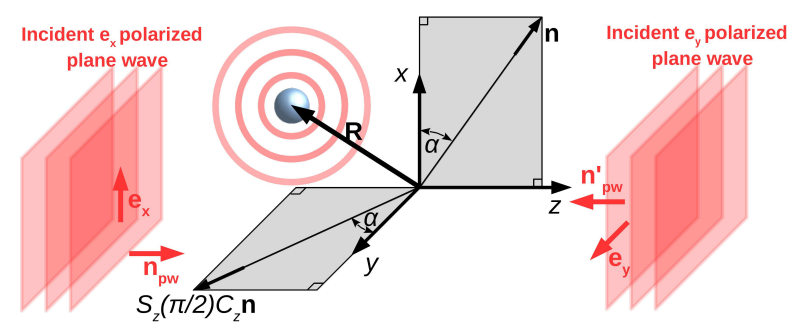

Fig. 2. Two counterpropagating plane waves are scattered on the particle; $\boldsymbol{n}_{\mathrm{pw}}$ and $\boldsymbol{n}_{\mathrm{pw}}^{\prime}$ define the directions of their propagation, $\boldsymbol{n}$ and $S_{\frac{\pi}{2}} C_{z} \boldsymbol{n}$ are unit vectors, which define the directions of observation, $\cos \alpha=n_{x}$

waves, it is possible to select the directions, the phase difference between which depends on $R_{z}$ and doesn't depend on the properties of a particle.

Consider the scattering of the plane wave propagating in the direction opposite to the polarization along the axis $y$. The scattered far field can be expressed as

$\boldsymbol{F}^{\prime}(\boldsymbol{n}, \boldsymbol{R})=C_{z} S_{\frac{\pi}{2}} \boldsymbol{F}\left(S_{\frac{\pi}{2}}^{T} C_{z} \boldsymbol{n}, S_{\frac{\pi}{2}}^{T} C_{z} \boldsymbol{R}\right)$,

where

$C_{z}=\left(\begin{array}{ccc}1 & 0 & 0 \\ 0 & 1 & 0 \\ 0 & 0 & -1\end{array}\right)$,

$S_{\frac{\pi}{2}}=\left(\begin{array}{ccc}0 & -1 & 0 \\ 1 & 0 & 0 \\ 0 & 0 & 1\end{array}\right)$,

$S_{\frac{\pi}{2}}$ is a matrix which defines the rotation by the angle $\frac{\pi}{2}$ around the $z$ axis, and $S_{\frac{\pi}{2}}^{T}$ is its transposed matrix. Using (5), we have

$$
\begin{aligned}
& \boldsymbol{F}^{\prime}(\boldsymbol{n}, \boldsymbol{R})=C_{z} S_{\frac{\pi}{2}} \exp \left(\mathrm{i} k\left[\boldsymbol{e}_{z}-S_{\frac{\pi}{2}}^{T} C_{z} \boldsymbol{n}\right] \times\right. \\
& \left.\times\left[S_{\frac{\pi}{2}}^{T} C_{z} \boldsymbol{R}-\boldsymbol{R}\right]\right) \boldsymbol{F}\left(S_{\frac{\pi}{2}}^{T} C_{z} \boldsymbol{n}, \boldsymbol{R}\right) .
\end{aligned}
$$

Let us consider the scattering of two plane waves that are propagating in the opposite directions and have orthogonal linear polarizations as shown in Fig. 2.

The total scattered far field

$$
\boldsymbol{F}^{\sigma}(\boldsymbol{n}, \boldsymbol{R})=\boldsymbol{F}^{\prime}(\boldsymbol{n}, \boldsymbol{R})+\boldsymbol{F}(\boldsymbol{n}, \boldsymbol{R})
$$

can be expressed through only the function $\boldsymbol{F}(\boldsymbol{n}, \boldsymbol{R})$, by using (21).

Let us consider only the directions $\boldsymbol{n} \perp \boldsymbol{e}_{x}$ or $\boldsymbol{n} \perp$ $\boldsymbol{e}_{y}$. According to (11) and (12), we have

$$
\begin{aligned}
& \boldsymbol{F}^{\sigma}(\boldsymbol{n}, \boldsymbol{R}) \boldsymbol{e}_{x}=\boldsymbol{F}(\boldsymbol{n}, \boldsymbol{R}) \boldsymbol{e}_{x} \\
& \boldsymbol{F}^{\sigma}(\boldsymbol{n}, \boldsymbol{R}) \boldsymbol{e}_{y}=\boldsymbol{F}^{\prime}(\boldsymbol{n}, \boldsymbol{R}) \boldsymbol{e}_{y}
\end{aligned}
$$


Using (21), we have finally:

$$
\boldsymbol{F}^{\sigma}\left(S_{\frac{\pi}{2}} C_{z} \boldsymbol{n}, \boldsymbol{R}\right) \boldsymbol{e}_{y}=\exp (\mathrm{i} \triangle \psi(\boldsymbol{n}, \boldsymbol{R})) \boldsymbol{F}^{\sigma}(\boldsymbol{n}, \boldsymbol{R}) \boldsymbol{e}_{x}
$$

The phase difference

$\triangle \psi(\boldsymbol{n}, \boldsymbol{R})=k\left[\left(n_{x}+n_{y}\right) R_{x}+\right.$

$\left.+\left(n_{y}-n_{x}\right) R_{y}-2\left(1-n_{z}\right) R_{z}\right]$.

As was written before, we consider only the directions with $n_{x}=0$ or $n_{y}=0$. So, expression (26) can be simplified a bit, but it contains, in general, all three coordinates of the scattering particle. Such expression can be used to determine $R_{z}$ of the particle, for example, in the case where $R_{x}$ and $R_{y}$ are already known.

\section{Discussion and Conclusions}

The consideration of the symmetry of the scattered wave in the case of the linearly polarized (say, $\boldsymbol{e}_{x}$ ) plane wave scattering on a microbead or Rayleighsized particle, which is placed at the origin leads to the following results.

Regardless of the particle position, the proper selection of a pair of directions, which are mutual reflections with respect to the $x z$ (or $y z$ ) plane results in the relation for the far-field complex amplitudes along these directions in the following way. Their electric field vector directions are the same mirror-reflected (with opposite sign in the case of $y z$ plane reflection), and the phase shift depends linearly on the particle coordinate $\boldsymbol{R}$ according to (13)-(15).

The fact of a linear dependence of the phase shift on the particle coordinate can be used for the development of a new-principle-based method of highsensitive particle detection. One such pair is enough for the determination of the $y$ - or $x$-position, respectively. But the determination area is limited, because the phase shift can be measured only in the $[-\pi, \pi)$ interval. The first limitation can be overcome, by using both types of direction pairs reflected to each other relative to the both $x z$ and $y z$ planes. Another issue can be overcome, by using more directions of observation or differing wavelengths of the incident wave.

According to the described principles, the detection of a particle position is possible by measuring the phase difference between the far-field components in selected directions. There are numerous ways for its realization. For example, it can be based on the measurement of Stokes parameters [17] or on phase shift interferometry [18].

In any case, if only one plane wave is used for the illumination it is possible to determine only the $x$ or $y$ position. Such problem can be resolved, by using two plane waves propagating in the directions opposite to the orthogonal polarization, as was considered in subsection (2.2). To make the measured phase shift independent of the particle parameters, the directions should be chosen in a different way. First of all, one observation direction should be orthogonal to the $x$ or $y$ axis. Another ones should be a reflection related to the $x y$ plane and a rotation around the $z$ axis of the first one. Another difference is that the phase shift is between the projections onto the $x$ and $y$ axis, but not for the total field, as in the case of single wave diffraction. Such statements are described in (23)-(25). The phase difference is described in (26), and it depends linearly on the coordinate of a particle as well. But the difference from the single wave scattering is that the phase shift can depend on the $z$ coordinate.

All such relations can be applied to the scattering on Rayleigh-sized particles and any particle with spherical symmetry, for example, on microbeads. All results are general, because all of them were derived, by using only symmetry rules.

For a wide range of applications, the sensitivity and short response time are critical. Nowadays, there are numerous light-based high-speed response sensitive particle position detection methods (for review, see [19]). However, all of them have some limitations. One of the limitations is a nonlinearity of such methods. Another one is that the behavior of a detector varies with a variation of the size or refractive index of a particle. So, the absolute axial position measurement becomes a challenging issue. In the present work, the relation between the phase shift and particle coordinates is linear. This means that the particle position detection based on the principles described in the present work can be not only one of the highspeed sensitive methods, but the first one of this class that measures the absolute axis position directly.

1. E.U. Condon, G.H. Shortley. The Theory of Atomic Spectra (Cambridge Univ. Press, 1951) [ISBN-13: 9780521092098, ISBN-10: 0521092094].

2. P. Michler. Single Quantum Dots: Fundamentals, Applications, and New Concepts, Topics in Applied Physics (Springer, 2003) [ISBN: 978-3-540-39180-7].

ISSN 2071-0194. Ukr. J. Phys. 2017. Vol. 62, No. 2 
3. C.F. Bohren, D.R. Huffman. Absorption and Scattering of Light by Small Particles (Wiley-VCH, 1998) [ISBN-13: 978-0471293408, ISBN-10: 0471293407].

4. G. Gouesbet, G. Gréhan. Generalized Lorenz-Mie Theories (Springer, 2011) [DOI: 10.1007/978-3-642-17194-9].

5. J.A. Lock, G. Gouesbet. Generalized Lorenz-Mie theory and applications. J. Quant. Spectr. Radiat. Transfer 110, 800 (2009) [DOI: 10.1016/j.jqsrt.2008.11.013].

6. T. Wriedt. Mie theory: a review. The Mie theory. Basics and applications. W. Hergert, T. Wolfram (eds.) (Springer, 2012), p. 53 [DOI: 10.1007/978-3-642-28738-1_2].

7. A. Heifetz, S.-C. Kong, A.V. Sahakian, A. Taflove, V. Backman. Photonic nanojets. J. Comput. Theor. Nanosci. 6, 1979 (2009) [DOI: DOI: 10.1166/ jctn.2009.1254].

8. A.D. Kiselev, D.O. Plutenko. Mie scattering of LaguerreGaussian beams: Photonic nanojets and near-field optical vortices. Phys. Rev. A 89, 043803 (2014) [DOI: 10.1103/PhysRevA.89.043803].

9. A.M. Shakhov, A.A. Astafiev, D.O. Plutenko, O.M. Sarkisov, A.I. Shushin, V.A. Nadtochenko. Femtosecond optical trap-assisted nanopatterning through microspheres by a single Ti:sapphire oscillator. J. Phys. Chem. C 119, 12562 (2015) [DOI: 10.1021/acs.jpcc.5b00478].

10. P. Ferrand, J. Wenger, A. Devilez, M. Pianta, B. Stout, N. Bonod, E. Popov, H. Rigneault. Direct imaging of photonic nanojets. Opt. Express 16, 6930 (2008) [DOI: 10.1364/OE.16.006930].

11. M.-S. Kim, T. Scharf, S. Mühlig, C. Rockstuhl, H.P. Herzig. Engineering photonic nanojets. Opt. Express 19, 10206 (2011) [DOI: 10.1364/OE.19.010206].

12. A.D. Kiselev, D.O. Plutenko. Optical trapping by Laguerre-Gaussian beams: Far-field matching, equilibria, and dynamics. Phys. Rev. A 94, 013804 (2016) [DOI: 10.1103/ PhysRevA.94.013804].

13. A.D. Kiselev, D.O. Plutenko. Mie scattering of LaguerreGaussian beams: Photonic nanojets and near-field optical vortices. Phys. Rev. A 89, 043803 (2014) [DOI: 10.1103/PhysRevA.89.043803].
14. A. Kiselev, D. Plutenko. Light scattering of LaguerreGaussian beams: near-field structures and symmetries. Nanosystems: Physics, Chemistry, Mathematics 7, 349 (2016) [DOI: 10.17586/2220-8054-2016-7-2-349-370].

15. M. Mishchenko, L. Travis, A. Lacis. Scattering, Absorption, and Emission of Light by Small Particles (Cambridge Univ. Press, 2002) [ISBN 9780521782524].

16. J.P. Barton. Electromagnetic field calculations for a sphere illuminated by a higher-order Gaussian beam. II. Far-field scattering. Appl. Opt. 37, 3339 (1998) [DOI: 10.1364/ AO.37.003339].

17. V.G. Denisenko, A. Minovich, A.S. Desyatnikov, W. Krolikowski, M.S. Soskin, Y.S. Kivshar. Mapping phases of singular scalar light fields. Opt. Lett. 33, 89 (2008) [DOI: 10.1364/OL.33.000089].

18. G. Lai, T. Yatagai. Generalized phase-shifting interferometry. J. Opt. Soc. Am. A 8, 822 (1991) [DOI: 10.1364/ JOSAA.8.000822].

19. K.C. Neuman, S.M. Block. Optical trapping. Rev. Sci. Instrum. 75, 2787 (2004) [DOI: 10.1063/1.1785844].

Received 11.07.16

Д.О. Плутенко, М.В. Васнєцов

СИМЕТРІЯ РОЗСІЯНОГО

НА СФЕРИЧНІЙ ЧАСТИНЦІ СВІТЛА ТА ФАЗОВИЙ

ЗСУВ, ІНДУКОВАНИЙ ЇЇ ТРАНСЛЯЦІЄЮ

$\mathrm{P}$ е $з$ ю м е

Розглядається дифракція плоскої хвилі, а також суперпозиція розсіяння від двух зустрічних ортогонально поляризованих плоских хвиль на сферичній частинці. Обговорюється вплив трансляції частинки на комплексну амплітуду поля в дальній зоні хвилі розсіяння. Показана можливість вибору симетричних відносно один одного напрямів спостереження, для яких фазовий зсув розсіяної хвилі залежить тільки від координати частинки. Всі висновки носять загальний характер, оскільки засновані лише на міркуваннях симетріі. Результати можуть бути корисні для розробки нового методу детектування положення частинки з високою чутливістю та швидкодією. 